\title{
CORRECTIONS
}

\section{The management of dry eye}

This Clinical Review from Drug and Therapeutics Bulletin (BMJ 2016;353:i2333, doi:10.1136/bmj.i2333) contains an error in the section on "Other treatments for dry eye." The "insertion of punctal plugs," which is mentioned as a treatment in the third paragraph, is used "to block the lacrimal drainage system" [not "to block the lacrimal glands" as was published]. 